\title{
Locating Datum Modeling for Minimum Normal Error in Aircraft Digital Assembly System
}

\author{
Yulong $\mathrm{Hu}$, Zhongqi Wang, Cong Ren and Xining Li
}

The Key Laboratory of Contemporary Design and Integrated Manufacturing Technology, Northwestern Polytechnical University, Xi'an, 710072, China

Keywords:Locating datum modeling, Aircraft digital assembly, Automatic assembly positioning.

\begin{abstract}
This paper analyzes the status of aircraft digital assembly process locating datum modeling methods, study the localization process of the aircraft digital assembly. By analyzing the error evaluation criteria for assembly positioning, proposed a locating datum modeling method for the minimum normal error, and the locating datum model can get the targeting points of aircraft digital assembly process. Finally, through the digital assembly locating datum modeling of one aircraft to verify, and get a better results.
\end{abstract}

\section{Introduction}

With the use of digital assembly tooling, not only improve the efficiency of aircraft assembly industry, but also reduces the number of tooling, because of its flexibility can be applied to the assembly of different components. In this case, the use of modern standard equipment such as standard robots, PC-computers and a newly developed spatial sensor system for precision measurements of positions [1]. For different mounting target, digital aircraft assembly tooling need to adapt to dynamic assembly environments, digital measuring device for measuring a benchmark during assembly gradually moving generally $[2,3]$. Digital measuring instruments relied upon in the traditional sense, the calculation method of measurement results also tend to use the least squares method and weighted least squares method [4, 5]. These methods can be used for general reference solving, but it is difficult to meet the positioning error criterion in assembly areas.

The positioning process of digital assembly for aircraft is shown in Fig.1.

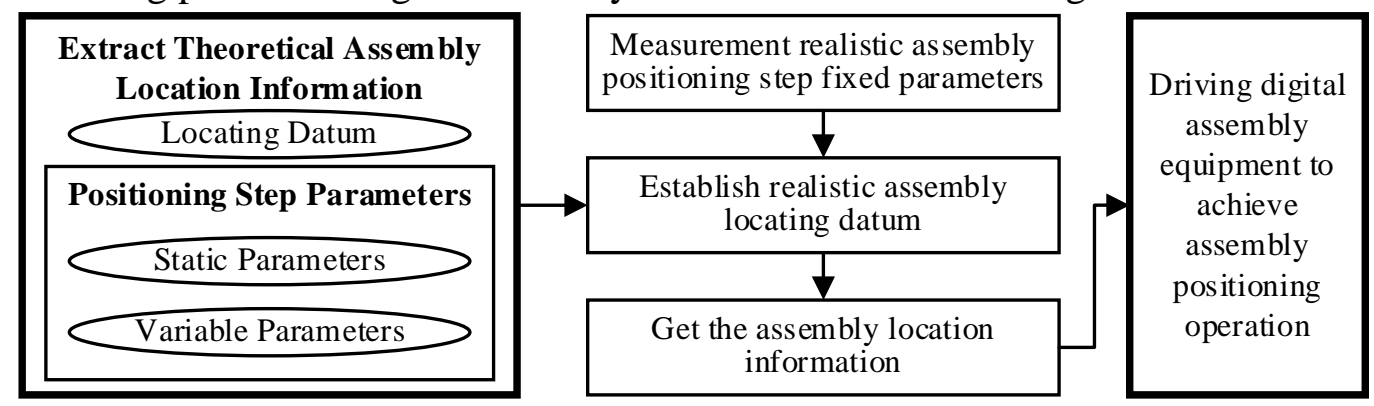

Fig.1: The positioning process of digital assembly

\section{Problem Definition and Modeling Process}

During the assembly of the aircraft, technology datum by function into locating datum, assembly datum, measuring datum and hybrid datum. Locating datum refers to the benchmark used to determine the relative position of the structures on the equipment or process equipment [6].

In a unified world coordinate system, locating datum modeling of digital assembly for aircraft mainly related to the Theoretical Assembly Environment (TAE) and Actual Assembly Environment (AAE) into two parts. Assembly locating datum is expressed as $\mathrm{AD}$, and positioning step parameters includes static parameters (SP) and variable parameters (VP), it is shown in Fig.2. Generally, it is known ADTAE, SPTAE, VPTAE and SPAAE, the process of solving ADAAE is called assembly locating datum modeling, and then it is known ADAAE, the process of solving VPAAE is called positioning step parameter solution. 


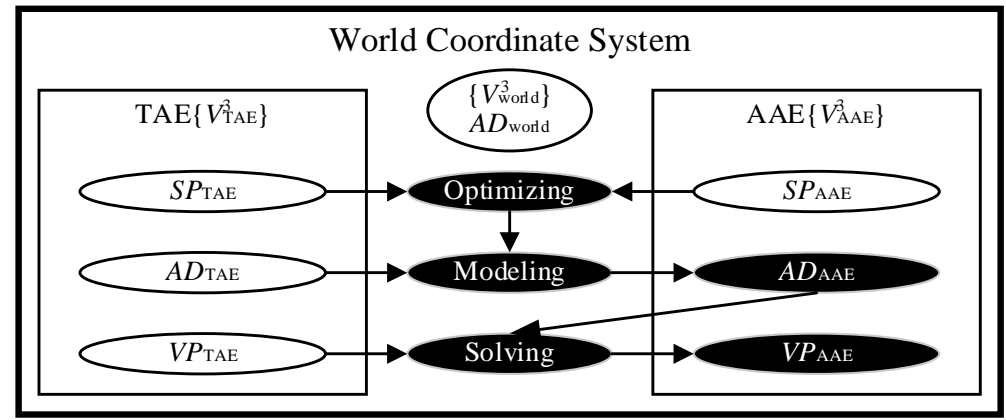

Fig.2: Locating datum modeling of aircraft digital assembly

\section{Assembly Locating Datum Modeling}

\section{Performance Analysis.}

Shape error is the amount of change for the actual shape of the measured elements relative to its ideal elements, and an ideal location factors should meet the minimum criteria. The so-called minimum condition, refers to the maximum amount of fluctuation of its ideal actual measured feature factor is minimum. In this case, the shape and the tolerance band are the same, the actual measured feature containment area is minimized, the minimum width or diameter of the inclusion region is the actual measured shape error elements. For contour elements, meet the minimum conditions of ideal elements located outside entity and parts in contact with the actual measured elements, and the maximum amount of change in the measured elements of the ideal elements to a minimum, for locating surface is its normal maximum error amount of change in the minimum, is shown in Fig.3.

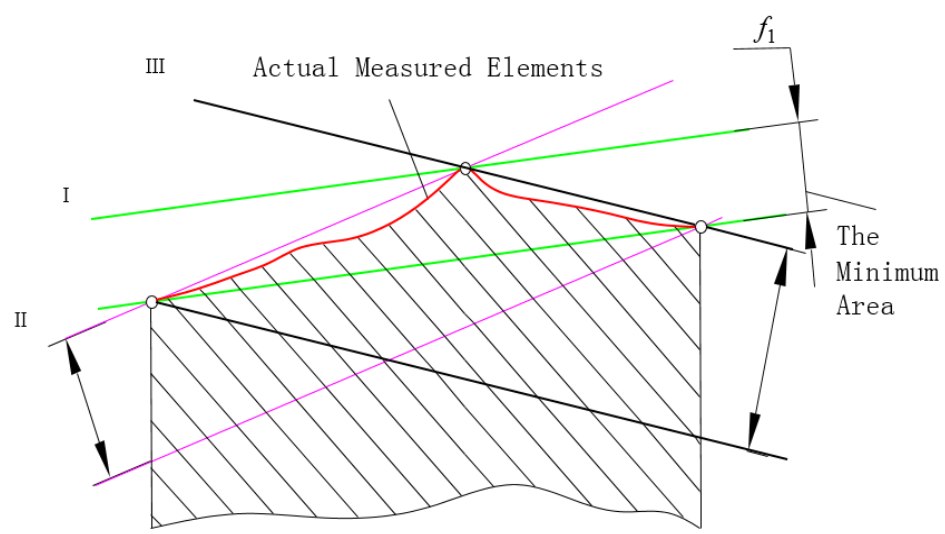

Design Variables.

Fig.3: The minimum conditions outline elements

The design variables include locating datum statements and positioning step parameter. The locating datum is expressed as $\mathrm{O}=[\mathrm{a}, \mathrm{b}, \mathrm{c}] \mathrm{T}$ and $\mathrm{Ro}=[\alpha, \beta, \gamma] \mathrm{T}$. The locating datum $A D_{\text {TAE }}^{\text {world }}$ in World Coordinate System is

$$
A D_{\text {TAR }}^{\text {world }}=\left\{\begin{array}{l}
\text { world } T=\operatorname{Trans}(a, b, c) \operatorname{Rot}(x, \alpha) \operatorname{Rot}(y, \beta) \operatorname{Rot}(z, \gamma) \\
\left.\left[\begin{array}{llll}
1 & 0 & 0 & a \\
0 & 1 & 0 & b \\
0 & 0 & 1 & c \\
0 & 0 & 0 & 1
\end{array}\right]\left[\begin{array}{cccc}
1 & 0 & 0 & 0 \\
0 & \cos \alpha & -\sin \alpha & 0 \\
0 & \sin \alpha & \cos \alpha & 0 \\
0 & 0 & 0 & 1
\end{array}\right]\left[\begin{array}{cccc}
\cos \beta & 0 & \sin \beta & 0 \\
0 & 1 & 0 & 0 \\
-\sin \beta & 0 & \cos \beta & 0 \\
0 & 0 & 0 & 1
\end{array}\right]\left[\begin{array}{cccc}
\cos \gamma & -\sin \gamma & 0 & 0 \\
\sin \gamma & \cos \gamma & 0 & 0 \\
0 & 0 & 1 & 0 \\
0 & 0 & 0 & 1
\end{array}\right]\right\}
\end{array}\right.
$$

For the space of a locating surface, the existence of the equation $A x+B y+C z+1=0$, expressed as $S_{T A E}=[A, B, C]^{\mathrm{T}}$. In the positioning step parameters, there are $N$ point and $M$ surface in $S P$, there are $L$ point in VP. $S P$ TAE is

$S P_{A A E}$ is

$$
S P_{\text {TAE }}=\left\{\begin{array}{l}
P_{\text {TAE }}=\left[\left(P_{T A E}\right)_{1} \cdots\left(P_{T A E}\right)_{i} \cdots\left(P_{T A E}\right)_{N}\right], i \in[1, N], i \in N^{+} \text {and }\left(P_{T A E}\right)_{i}=\left[\left(x_{T A E}\right)_{i},\left(y_{T A E}\right)_{i},\left(z_{T A E}\right)_{i}\right]^{T} \\
S_{\text {TAE }}=\left[\left(S_{T A E}\right)_{1} \cdots\left(S_{T A E}\right)_{j} \cdots\left(S_{T A E}\right)_{M}\right], j \in[1, M], j \in N^{+} \text {and }\left(S_{T A E}\right)_{j}=\left[A_{j}, B_{j}, C_{j}\right]^{T}
\end{array}\right.
$$




$$
S P_{A A E}=\left\{\begin{array}{l}
P_{A A E}=\left[\left(P_{A A E}\right)_{1} \cdots\left(P_{A E E}\right)_{i} \cdots\left(P_{A A E}\right)_{N}\right], i \in[1, N], i \in N^{+} \text {and }\left(P_{A A E}\right)_{i}=\left[\left(x_{A E E}\right)_{i},\left(y_{A A E}\right)_{i},\left(z_{A A E}\right)_{i}\right]^{T} \\
S_{A E E}=\left[\left(S_{A E}\right)_{1} \cdots\left(S_{A E E}\right)_{j} \cdots\left(S_{A A E}\right)_{M}\right], j \in[1, M], j \in N^{+} \text {and }\left(S_{A A E}\right)_{j}=\left[\left(s_{A A E}\right)_{j 1} \cdots\left(s_{A E}\right)_{j d} \cdots\left(s_{A A E}\right)_{j D}\right]
\end{array}\right.
$$

In the Eq.3, $d \in\left[1, D_{j}\right], d \in N^{+}, D_{j}$ is number of discrete points in the $j$-th of the locating surface. $V P_{T A E}$ and $V P_{A A E}$ are

$$
\left\{\begin{array}{l}
V P_{T A E}=\left[\left(V P_{T A E}\right)_{1} \cdots\left(V P_{T A E}\right)_{k} \cdots\left(V P_{T A E}\right)_{L}\right], k \in[1, L], k \in N^{+} \text {and }\left(V P_{T A E}\right)_{k}=\left[\left(x_{\text {TAEV }}\right)_{k},\left(y_{\text {TAEV }}\right)_{k},\left(z_{\text {TAEV }}\right)_{k}\right]^{T} \\
V P_{\text {AAE }}=\left[\left(V P_{A A E}\right)_{1} \cdots\left(V P_{A E E}\right)_{k} \cdots\left(V P_{A A E}\right)_{L}\right], k \in[1, L], k \in N^{+} \text {and }\left(V P_{A A E}\right)_{k}=\left[\left(x_{A A E V}\right)_{k},\left(y_{A A E V}\right)_{k},\left(z_{A A E V}\right)_{k}\right]^{T}
\end{array}\right.
$$

Locating surface location error limit is $E s=\left[E s_{1}, E s_{2}, \cdots E s_{j} \cdots, E s_{M}\right], j \in[1, M], j \in N^{+}$.

Locating point location error limit is $E p=\left[E p_{1}, E p_{2}, \cdots E p_{i} \cdots, E p_{N}\right], i \in[1, N], i \in N^{+}$.

Objective Function and Constraint Conditions.

To solve ( $\mathrm{a}, \mathrm{b}, \mathrm{c}, \alpha, \beta, \gamma)$, the minimum objective function is

$$
\begin{aligned}
& \min f(a, b, c, \alpha, \beta, \gamma)=\min \left\{\max \left[\left(\bigcup_{j=1}^{M} \frac{\Delta s_{j}}{E s_{j}}\right) \cup\left(\bigcup_{i=1}^{N} \frac{\Delta p_{i}}{E p_{i}}\right)\right]\right\}
\end{aligned}
$$

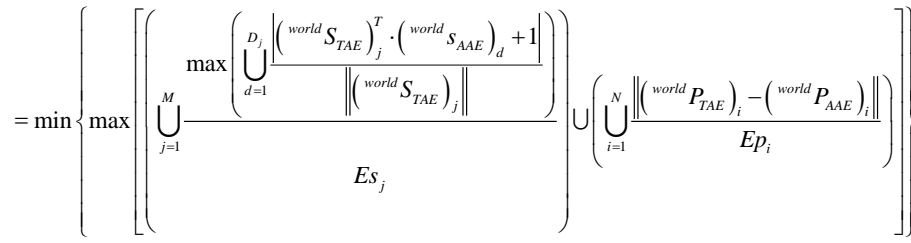

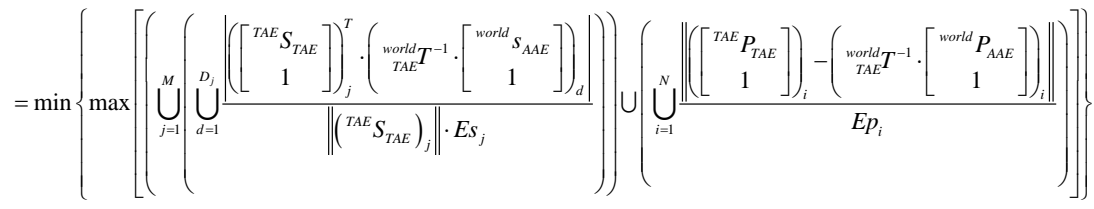

The constraint conditions are

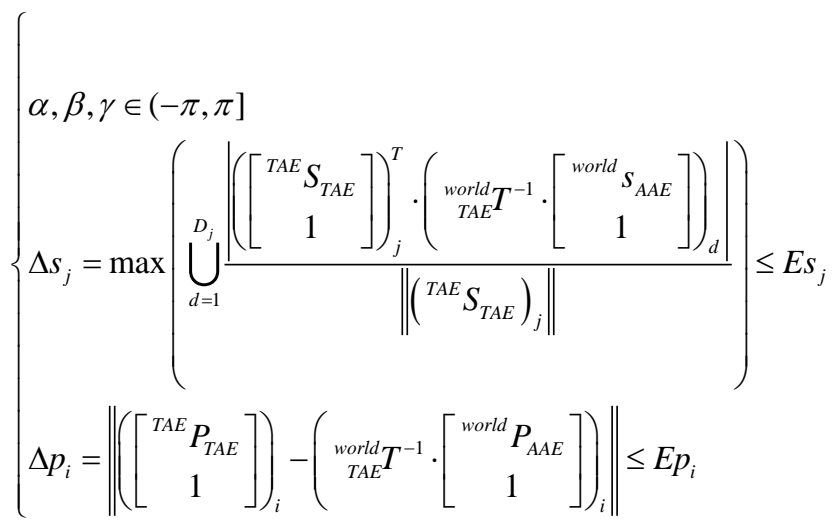

The swarm intelligenc could be used to solve this problem. For example, the particle swarm optimization (PSO) and so on [7, 8].

\section{Positioning Step Parameter Solution.}

When ${ }^{\text {world }} T$ has been obtained, the VPAAE is

$$
\left[\begin{array}{c}
\text { world } V P_{A A E} \\
1
\end{array}\right]={ }_{\text {TAE }}^{\text {worl }} T \cdot\left[\begin{array}{c}
{ }^{T A E} V P_{T A E} \\
1
\end{array}\right]
$$

\section{Computational examples and analysis}

Tested through digital flexible assembly tooling for certain type of aircraft, tooling and assembly object is shown in Fig.4. 

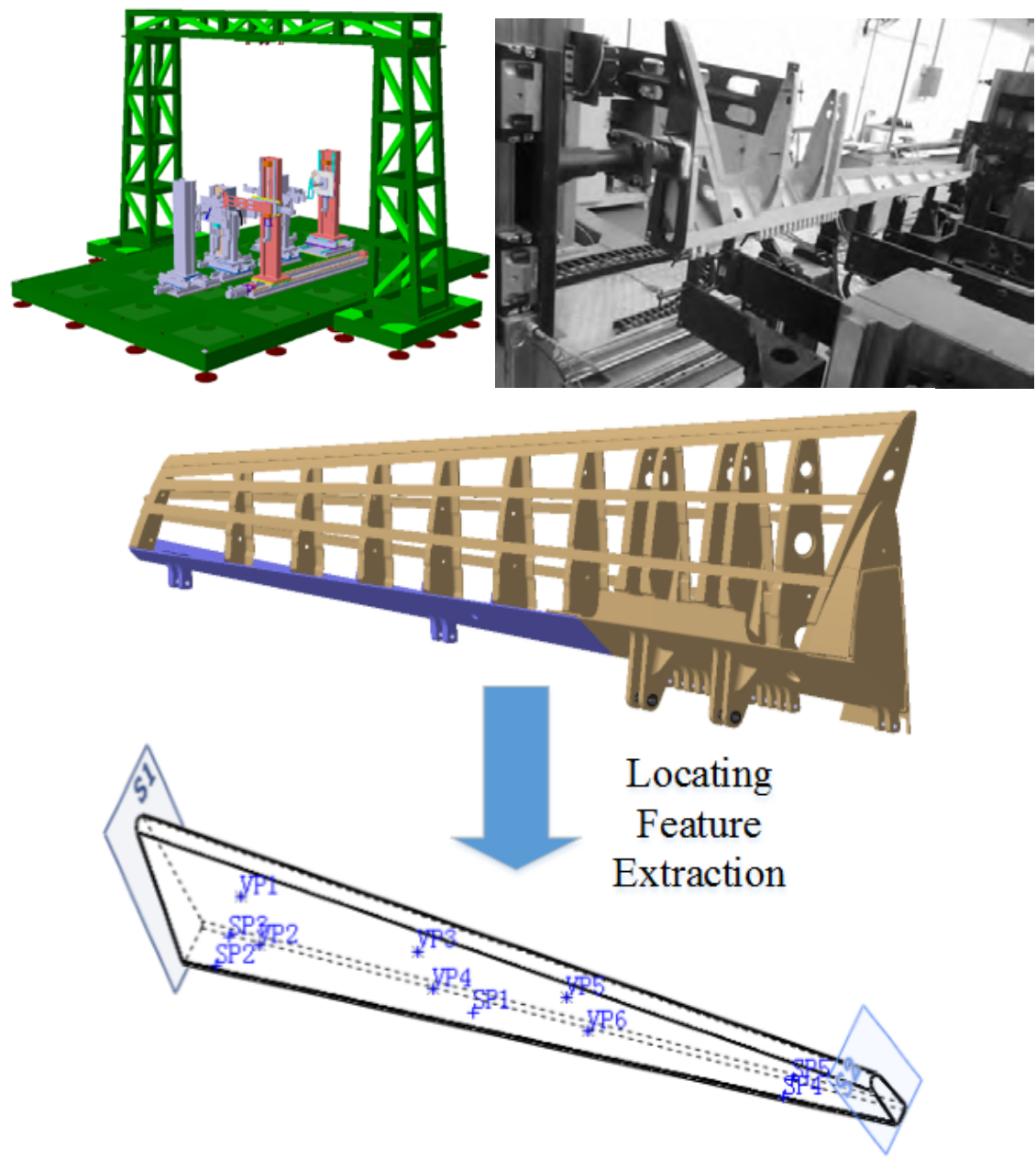

Fig.4: Tooling and assembly object for testing Known parameters $(M=2, N=5, L=6, D=[6,6]$. Unit is $\mathrm{m})$ is

$$
\begin{aligned}
& S_{\text {TAE }}=\left[\begin{array}{ll}
\left(S_{\text {TAE }}\right)_{1} & \left(S_{\text {TAE }}\right)_{2}
\end{array}\right]=\left[\begin{array}{cc}
-1.4635459 & -0.3295303 \\
-0.89019 & -0.34616 \\
-0.27919 & -0.09585
\end{array}\right] \\
& P_{\text {TAE }}=\left[\begin{array}{lllll}
\left(P_{T A E}\right)_{1} & \left(P_{T A E}\right)_{2} & \left(P_{T A E}\right)_{3} & \left(P_{T A E}\right)_{4} & \left(P_{T A E}\right)_{5}
\end{array}\right] \\
& =\left[\begin{array}{lllll}
1.114584 & 0.560135 & 0.564271 & 1.807423 & 1.810008 \\
0.134638 & -0.09017 & -0.07253 & 0.400538 & 0.411562 \\
1.393234 & 1.353984 & 1.276063 & 1.515865 & 1.467164
\end{array}\right] \\
& V P_{T A E}=\left[\begin{array}{llllll}
\left(V P_{T A E}\right)_{1} & \left(V P_{T A E}\right)_{2} & \left(V P_{T A E}\right)_{3} & \left(V P_{T A E}\right)_{4} & \left(V P_{T A E}\right)_{5} & \left(V P_{T A E}\right)_{6}
\end{array}\right]
\end{aligned}
$$

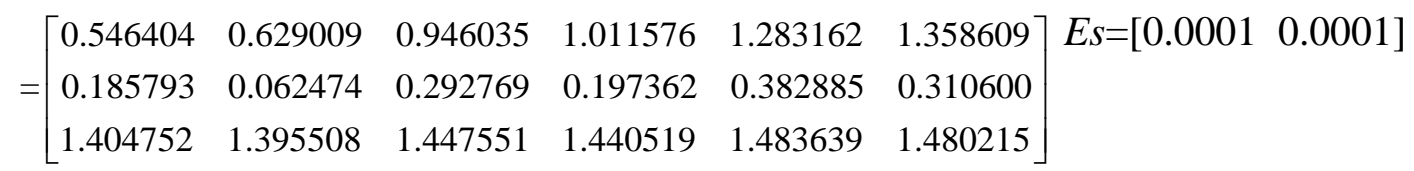

$$
\begin{aligned}
& E p=\left[\begin{array}{lllll}
0.0001 & 0.0001 & 0.0001 & 0.0001 & 0.0001
\end{array}\right]
\end{aligned}
$$

Measurement parameters is 


$$
\begin{aligned}
& S_{\text {AAE }}=\left[\begin{array}{ll}
\left(S_{A A E}\right)_{1} & \left(S_{A A E}\right)_{2}
\end{array}\right] \\
& =\left\{\begin{array}{l}
\left(S_{\text {AAE }}\right)_{1}=\left[\begin{array}{llllll}
\left(s_{\text {AAE }}\right)_{11} & \left(s_{A A E}\right)_{12} & \left(s_{A A E}\right)_{13} & \left(s_{\text {AAE }}\right)_{14} & \left(s_{\text {AAE }}\right)_{15} & \left(s_{A A E}\right)_{16}
\end{array}\right] \\
=\left[\begin{array}{lllllll}
1.866894 & 1.870807 & 1.943824 & 1.931619 & 1.977183 & 2.047761 \\
2.386466 & 2.357979 & 2.338947 & 2.299447 & 2.270955 & 2.276914 \\
2.996431 & 3.061442 & 3.125726 & 3.210626 & 3.287994 & 3.295397
\end{array}\right] \\
\left(S_{\text {AAE }}\right)_{2}=\left[\begin{array}{llllll}
\left(s_{\text {AAE }}\right)_{21} & \left(s_{\text {AAE }}\right)_{22} & \left(s_{\text {AAE }}\right)_{23} & \left(s_{\text {AAE }}\right)_{24} & \left(s_{\text {AAEE }}\right)_{25} & \left(s_{\text {AAE }}\right)_{26}
\end{array}\right] \\
=\left[\begin{array}{llllll}
1.898218 & 1.918082 & 1.906847 & 1.928432 & 1.916331 & 1.948395 \\
3.677213 & 3.678265 & 3.669398 & 3.672375 & 3.665435 & 3.673670 \\
4.161700 & 4.181714 & 4.217036 & 4.228220 & 4.251807 & 4.246631
\end{array}\right]
\end{array}\right. \\
& P_{A A E}=\left[\begin{array}{lllll}
\left(P_{A A E}\right)_{1} & \left(P_{A A E}\right)_{2} & \left(P_{A A E}\right)_{3} & \left(P_{A A E}\right)_{4} & \left(P_{A A E}\right)_{5}
\end{array}\right] \\
& =\left[\begin{array}{lllll}
1.986950 & 1.979383 & 2.052021 & 1.928122 & 1.973501 \\
2.812536 & 2.301967 & 2.323093 & 3.434231 & 3.447449 \\
3.700639 & 3.386432 & 3.360416 & 4.119920 & 4.103715
\end{array}\right]
\end{aligned}
$$

The results of assembly locating datum modeling is

$$
\begin{aligned}
& \min f(a, b, c, \alpha, \beta, \gamma)=6.20 \times 10^{-5}
\end{aligned}
$$

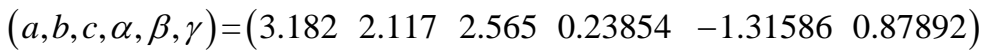

$$
\begin{aligned}
& \underset{\text { warld }}{\text { TAE }}=\left[\begin{array}{cccc}
0.160889 & -0.194194 & -0.967679 & 3.182000 \\
0.602372 & 0.795988 & -0.059587 & 2.117000 \\
0.781832 & -0.573316 & 0.245042 & 2.565000 \\
0 & 0 & 0 & 1
\end{array}\right]
\end{aligned}
$$

The results of positioning step parameter solution is

$$
{ }^{\text {world }} V P_{\text {AAE }}=\left[\begin{array}{llllll}
1.874480 & 1.920664 & 1.876587 & 1.912464 & 1.878405 & 1.907894 \\
2.510322 & 2.462472 & 2.833650 & 2.797606 & 3.106307 & 3.094420 \\
3.229902 & 3.362921 & 3.491503 & 3.595721 & 3.712258 & 3.811848
\end{array}\right]
$$

\section{Conclusions}

We expect that the work we propose here, when added to the work we have carried out so far, will lead to the advancement of aircraft digital assembly location research. The locating datum will allow more automated for digital assembly tooling and support incremental advances in the field.

\section{References}

[1] Kihlman H, Ossbahr G, Engström M, et al. Low-cost automation for aircraft assembly[R]. SAE Technical Paper, 2004.

[2] Millar A, Kihlman H. Reconfigurable flexible tooling for aerospace wing assembly[R]. SAE Technical Paper, 2009.

[3] Junbai L, Kai Z. Multi-point location theory, method, and application for flexible tooling system in aircraft manufacturing[J]. The International Journal of Advanced Manufacturing Technology, 2011, 54(5-8): 729-736.

[4] Tian S, Lu W, Tian H, et al. Comparing evaluated precision of straightness error among two spot, least square method and minimum envelope zone method[C]//Geoscience and Remote Sensing (IITA-GRS), 2010 Second IITA International Conference on. IEEE, 2010, 2: 67-70.

[5] Wang C, Qi F, Shi G, et al. A linear combination-based weighted least square approach for target localization with noisy range measurements[J]. Signal Processing, 2014, 94: 202-211.

[6] Mei, S. H. E. N., and HE Xiaozhao ZHANG Tiechang. "Assembly Modelling Based on Assemby Feature." China Mechanical Engineering 9 (2001): 016.

[7] Noel, Mathew M. "A new gradient based particle swarm optimization algorithm for accurate computation of global minimum." Applied Soft Computing 12.1 (2012): 353-359. 
[8] Tasgetiren, M. Fatih, et al. "Particle swarm optimization algorithm for makespan and maximum lateness minimization in permutation flowshop sequencing problem." Proceedings of the fourth international symposium on intelligent manufacturing systems, Sakarya, Turkey. 2004. 